\title{
The influence of microstructure on mechanical properties of SLM 3D printed Ti- 6Al-4V
}

\author{
Cecilie V. Funch ${ }^{1}{ }^{2}$, Kinga Somlo ${ }^{1}$, Konstantinos Poulios ${ }^{1}$, Sankhya Mohanty ${ }^{1}$, Marcel A.J. Somers ${ }^{1}$, Thomas L. Christiansen ${ }^{1}$ \\ ${ }^{1}$ Technical University of Denmark, Department of Mechanical Engineering \\ ${ }^{2}$ Corresponding author: cevfu@mek.dtu.dk
}

\begin{abstract}
This research focuses on establishing the relationship between the SLM process parameters, microstructure and mechanical properties in $3 \mathrm{D}$ printed ti-6Al-4V. To this end modelling of the thermal history, reflecting the applied process parameters, is linked to the as-printed microstructure, which, in turn, is linked to experimentally determined mechanical properties. The very high cooling rates in the centre of the specimen, as predicted by modelling, lead to a fine, martensitic microstructure, while the slower cooling rates close to the support structure lead to a fine lamellar $\alpha+\beta$ microstructure. The tensile properties, in particular the ductility, were found to depend on the printing orientation and surface finish.
\end{abstract}

\section{Introduction}

Titanium and its alloys have long been the materials of choice for medical and aerospace applications due to their unique combination of high strength, low density and, in medical, bio-compatibility. However, conventionally the areas of application have been limited by the high raw material cost. It has been shown that selective laser melting (SLM) is a cost effective route for producing titanium components for the aforementioned industries [1]. SLM is a near-net-shape additive manufacturing (AM) process, which reduces material waste by near-net shape manufacturing. Furthermore, the process enables a large degree of design freedom, customization and reduction of lead time [2].

Titanium Grade 5 (Ti-6Al-4V) accounts for the largest part of the world's titanium production due to the favourable combination of strength-to-weight ratio, good heat treatability and relatively high service temperatures. The material is very well-known from conventional manufacturing, however the unique thermal input from the SLM process results in the formation of non-equilibrium microstructures [6]. The local melting of the powder in the SLM process combined with the rapid movement of the laser results in steep temperature gradients and the formation of martensitic $\alpha$ ' phase inside elongated prior- $\beta$ grains $[3,4]$. This martensitic microstructure in printed parts has been shown to have a higher strength than in conventional Ti-6Al-4V, albeit much more brittle [4, 5, 6]. In most research, the ductility falls short of the $10 \%$ requirement stipulated in ASTM F2924 standard for additive manufacturing of Ti-6Al-4V [7] and a heat treatment is necessary for improving the mechanical properties.

SLM is a layer-based manufacturing process, which makes it necessary to consider also the build direction. Mezzetta et al. [6] found that tensile specimens printed in lying configuration have the lowest ductility and highest strength, as opposed to printing tensile specimens in standing geometry, which show the opposite relationship. Simonelli et al. [8] found that flat tensile bars lying on their side show improved ductility and strength. Therefore, it is important to understand the formation of the non-equilibrium microstructure and how it depends on the (local) cooling conditions as imposed by the printing parameters. This research focuses on the link between thermodynamics modelling of the heat input from the process to the resulting microstructure and mechanical performance.

\section{Materials and Methods}

The specimens used in this research were printed on a SLM500 printer in an Ar atmosphere, using titanium grade 23 powder from SLM Solutions, which is also known as a grade 5 ELI. It is alloyed with $6 \mathrm{wt} \% \mathrm{Al}$ and $4 \mathrm{wt} \% \mathrm{~V}$. A layer height of $60 \mu \mathrm{m}$ and a parallel stripes pattern strategy with $67^{\circ}$ rotation between layers was used. The most important build settings are found in Table 1 . Tensile bars with a length of $132 \mathrm{~mm}$ and a functional cross-section of $7 \times 6 \mathrm{~mm}^{2}$ were printed in three different orientations: horizontal, transverse and vertical. The bars were designed according to ASTM 8/8M. The tensile bars were machined to $6 \times 5 \mathrm{~mm}^{2}$ using electrical discharge machining (EDM). Some vertical specimens were printed with the final dimension and tested without additional machining. A stress relief treatment was carried out at $550{ }^{\circ} \mathrm{C}$ for 2 hours in $\mathrm{Ar}$ and a few selected cylindrical specimens of $\varnothing 10 \times 10 \mathrm{~mm}$ were removed prior to stress relief.

Table 1: Build parameters.

\begin{tabular}{||l|l|l|l||}
\hline & Speed $[\mathbf{m m} / \mathbf{s}]$ & Power $[\mathbf{W}]$ & Hatch distance $[\mathbf{m m}]$ \\
\hline Support & 650 & 150 & - \\
\hline Volume & 1100 & 350 & 0.120 \\
\hline Border & 650 & 150 & - \\
\hline Upskin - Remelting & 500 & 200 & 0.100 \\
\hline
\end{tabular}

(C) The Authors, published by EDP Sciences. This is an open access article distributed under the terms of the Creative Commons Attribution License 4.0 (http://creativecommons.org/licenses/by/4.0/). 
Micro hardness indentation was done using a load of $200 \mathrm{~g}$ and a dwell time of $10 \mathrm{~s}$. For tensile testing a MTS $312.21100 \mathrm{kN}$ servohydraulic tensile machine was used in displacement control mode. The cross head displacement rate was $0.05 \mathrm{~mm} / \mathrm{sec}$, the data acquisition rate was $100 \mathrm{~Hz}$. Strain measurements were conducted using an Instron extensometer with a gauge length of $12.5 \mathrm{~mm}$ and the results were evaluated according to ASTM 8/8M.The tensile bars were sectioned in three orthogonal cuts, the XY, XZ and YZ planes of the build plate respectively. Standard metallographic preparation was used and etching with Keller's reagent. Light optical microscopy (LOM) was conducted on an Olympus GX41, while scanning electron microscopy (SEM) was conducted on Zeiss Supra 35 FEGSEM (20 $\mathrm{kV}$, in-lens detector, $60 \mu \mathrm{m}$ aperture) and a Phenom Pro-X SEM. X-ray diffraction (XRD) was conducted on a Bruker D8 Advance diffractometer with $\mathrm{Cu}-\mathrm{K} \alpha$ radiation, using a locked-couple focusing principle and a $0.03{ }^{\circ} 2 \theta$ step size and measurement time of $8 \mathrm{~s}$ in the range $30-90^{\circ} 2 \theta$.

Macro-scale thermal simulations of the SLM process for the different tensile bars were performed using ANSYS Workbench. The in-built module for AM macro-simulation in ANSYS activates the material at its melting temperature to simulate the conversion of powder into a molten/solid domain, hence the material properties (e.g. the melting temperature, thermal conductivity, etc.) were artificially adjusted to produce maximum temperatures similar to a micro-scale simulation. The typical simulation in this study further added five layers ( 300 $\mu \mathrm{m})$ of material simultaneously, to achieve reasonable computational time within acceptable accuracy. A dwell time of 10s between layers was also included in the simulations corresponding to the time taken to process the other regions of the particular build and the time to lay down the new powder layers, neither are directly simulated.

\section{Results and Discussion}

The temperature history of the specimens is numerically modelled at two different locations: close to the support structure ( $\sim 300 \mu \mathrm{m}$ from the bottom) and at half the height of the specimen (not including the support structure). Due to the different orientations (horizontal, transverse and vertical) the half height location corresponds to $3 \mathrm{~mm}, 3.5 \mathrm{~mm}$ and $66 \mathrm{~mm}$ from the support structure, respectively. All measurements are from the centre of the build plane. The modelling results for the horizontal and vertical specimen are shown in Figure 1.

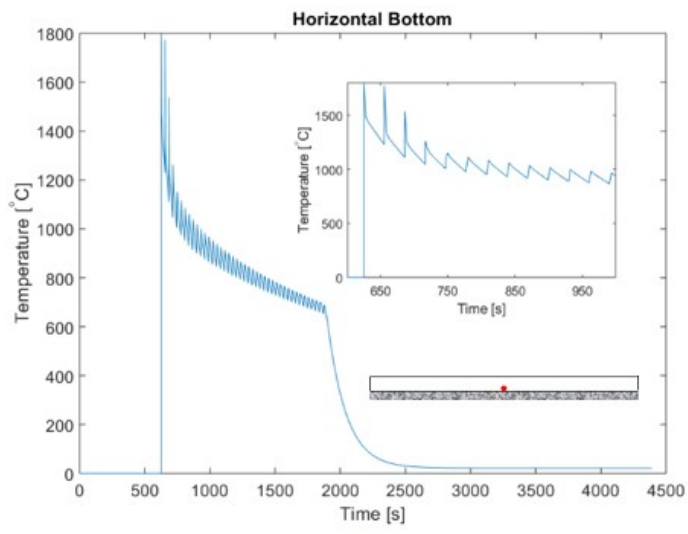

a)

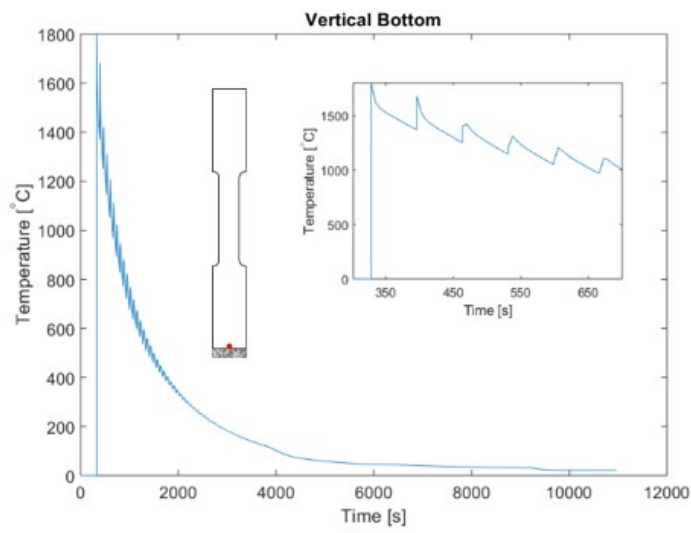

c)

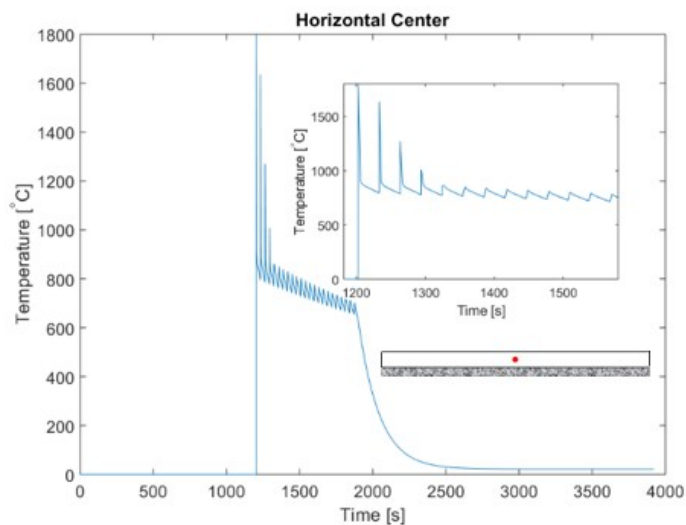

b)

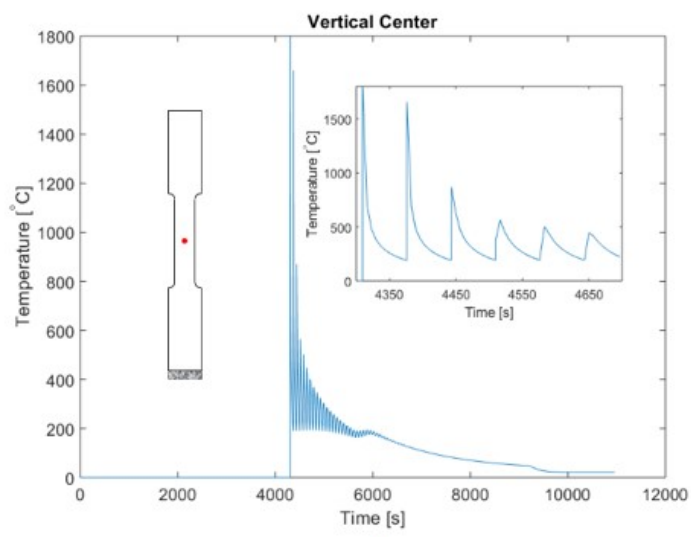

d)

Figure 1: Thermal history modelling results. Horizontal specimen: bottom (a) and centre (b). Vertical specimen: bottom (c) and centre (d).

The thermal history of the two specimens and locations is very different. The actual printing starts at $0 \mathrm{~s}$ and the temperature profile appears when the build reaches the investigated location. Melting occurs at the first sharp spike in temperature. Temperature cycling finishes when the print is completed and the part cools to room temperature. The horizontal bottom location is melted later than the vertical as its $\mathrm{XY}$ area is much larger and it requires longer time to print its support structure. For all orientations remelting occurs on printing the next layer, reflected as the second peak, which also exceeds the liquidus temperature $\left(1630{ }^{\circ} \mathrm{C}\right.$ for titanium grade 23$)$. During the third pass of the laser, the temperature is again raised, however it does not lead to (partial) melting. Here, the effective temperature at the reference point (the red dots in Fig. 1) will be within the $\beta$-phase region. Most interesting is the thermal behaviour between the $\beta$ - 
transus temperature $\left(977^{\circ} \mathrm{C}\right.$ ) and the martensite start temperature, Ms, around $825^{\circ} \mathrm{C}$. As seen in Figure 1 a) and c), cooling in this temperature range is rather slow in the bottom of both specimens as it is several hundreds of seconds before Ms is reached. Therefore, no martensite will form at this location. Considering the centre of the specimens, a dramatically different thermal history is observed. From the second cycle, the vertical specimen cools extremely fast through the $\beta$-transus to Ms temperature range. The third pass will exceed the Ms temperature again, but not reach $\beta$-transus, and, hence, potentially refines the microstructure. This short annealing step below the $\beta$ transus can also result in a slight partitioning of elements, causing an enrichment of V and Fe in $\beta$-phase, which causes a reduction in Ms. All subsequent laser passes do not raise the temperature sufficiently, but will effectively function as annealing (self-ageing) of the formed martensite. The repeated cycles in-between $\beta$-transus and Ms temperature make it very difficult to predict, which microstructure this thermal history results in. This means that it is of crucial importance to consider part geometry and its relation to thermal history and resulting microstructure.

The microstructure in the different orientations was evaluated after EDM, where the border, upskin and support microstructures were removed. The cuts along the $\mathrm{XZ}$ and $\mathrm{YZ}$ planes revealed identical microstructures (Figure $2 \mathrm{a}-\mathrm{c}$ ). Here prior- $\beta$ grains are visible, slightly elongated along the build direction. A series of bands parallel to the build plane is visible in the $\mathrm{XZ}$ plane. The distance between these bands is approximately $1-1.5 \mathrm{x}$ the layer thickness of $60 \mu \mathrm{m}$. This banding is observed under certain process conditions, however the cause for their formation is not clear. Thijs et al. [3] hypothesized that the bands reflect a segregation of $\mathrm{Al}$, causing the formation of a $\mathrm{Ti}_{3} \mathrm{Al}$ intermetallic phase corresponding to the layer height. These bands reflect anisotropy of the mechanical performance and therefore needs to be considered in the design stage. Possibly, a subsequent annealing step becomes necessary.

At higher magnifications (Figure $2 \mathrm{~b}$ ) and $-\mathrm{c}$ )) the martensitic microstructure is apparent, with each prior- $\beta$ grain containing multiple orientations of a' martensite. Looking in the build plane (XY), Figure $2 \mathrm{~d}-\mathrm{f}$ ), the grains appear more regular in shape, almost square in some regions. Again, the martensitic microstructure is obvious.
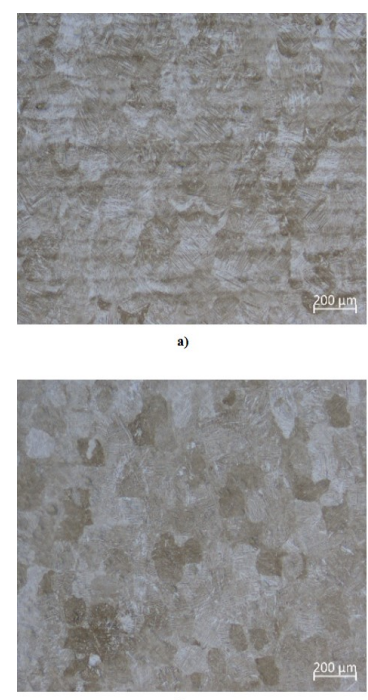

d)

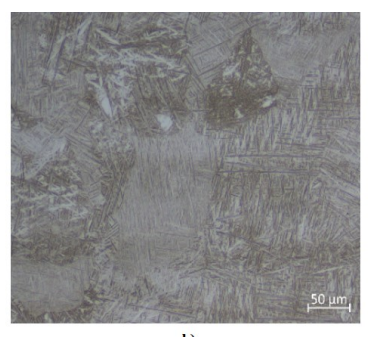

b)

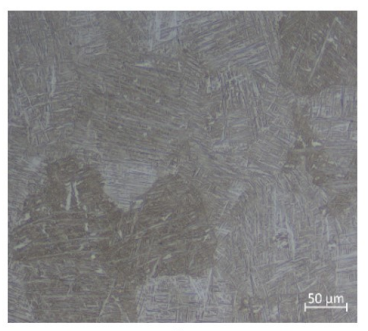

e)
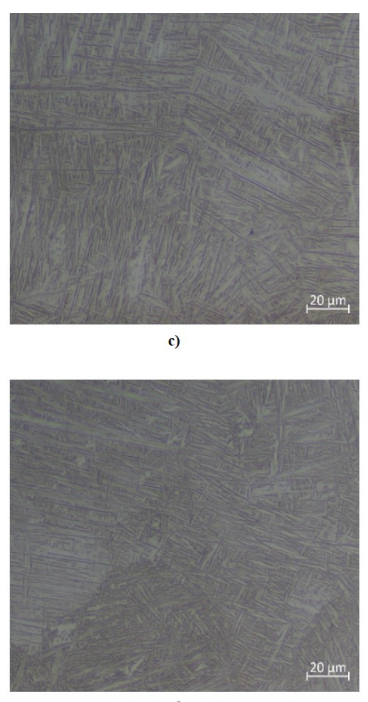

f)

Figure 2: LOM micrographs of vertical specimen. a), b) and c) XZ orientation. d), e) and f) XY orientation.

SEM allows to investigate the martensitic microstructure at higher resolution (Figure 3). Here, the different martensite morphologies are clearly visible. The primary martensite plates are the thickest with secondary and tertiary martensite forming between the primary resp. secondary needles. The width of the needles is generally small, the largest being around $\sim 500 \mathrm{~nm}$, which consistent with the reputation of the SLM process lead to fine martensitic microstructures.

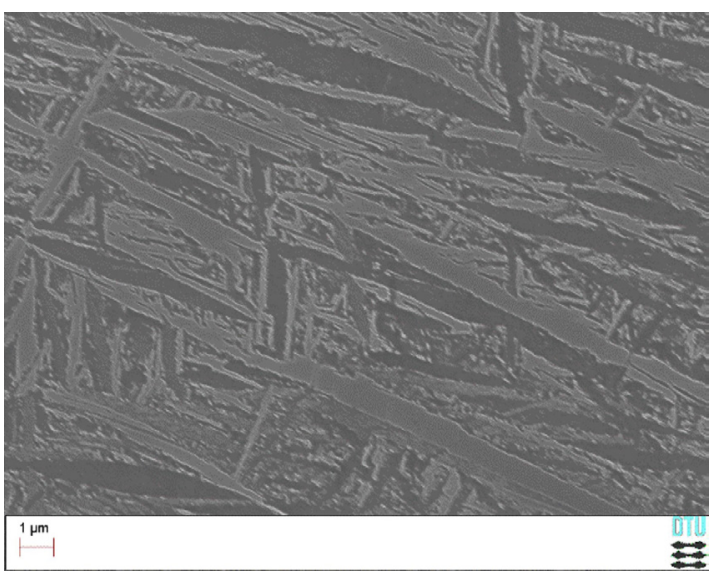

Figure 3: SEM micrograph of vertical specimen.

The microstructure is directly dependent on the cooling conditions following from the modelling of the temperature history of different locations. Therefore, it is necessary to investigate the regions, where the printing parameters, and therefore heat input, are changed, see 
Table 1. From Figure 4 is follows that the bottom location, close to where the support printing parameters were used, has a markedly different microstructure as compared to the bulk as seen in Figure 2. In the bottom, Figure $4 \mathrm{~d}$-f), the microstructure does not appear to be martensitic, but rather to consist of very fine lamellar $\alpha / \beta$. In addition, unmelted powder particles are present, next to where the support structure was located during the print. At the top, Figure $4 \mathrm{a}-\mathrm{c}$ ), the microstructure is similar to the bulk, (Figure 2 ). Here, elongated prior $\beta$ grains are visible and the martensitic microstructure is apparent, particularly in Figure $4 \mathrm{c}$ ). The main difference is in the banding, which is absent in the last $\sim 400 \mu \mathrm{m}$ of the build. This (lack of) feature indicates that the upskin remelting reaches this depth and as no further layers are deposited, no further banding appears.
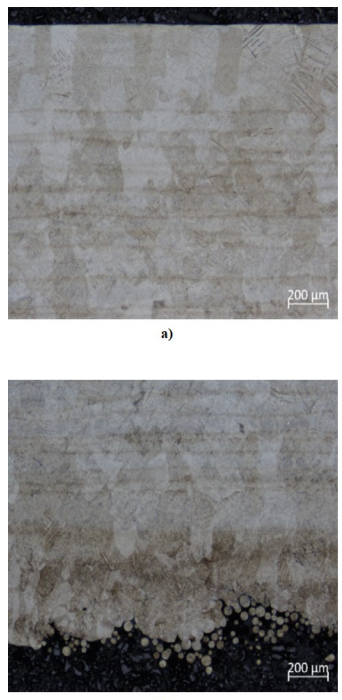
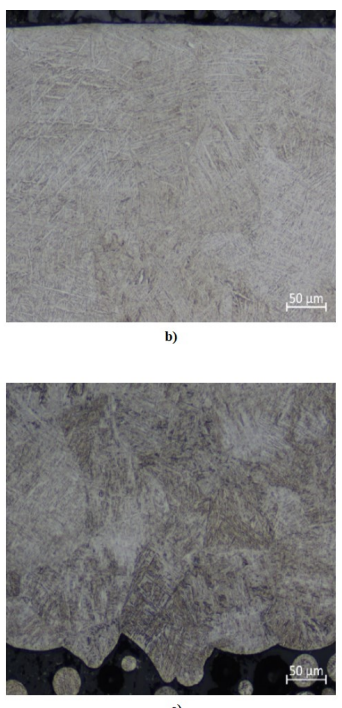
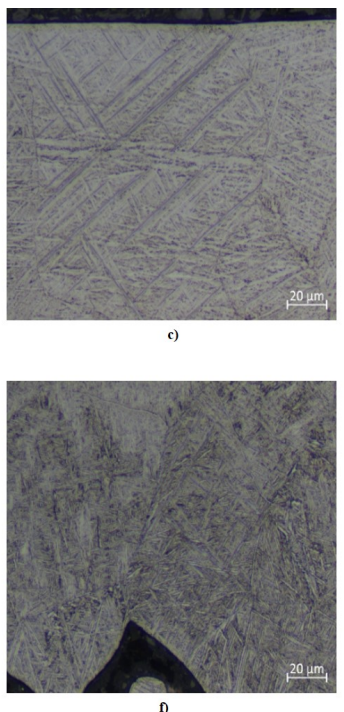

Figure 4: LOM micrographs of horizontal specimen at different locations. Top a), b), c) and Bottom d), e), f).

The micro hardness on all three orthogonal planes is shown in Figure 5. Due to the large standard deviation (spread) of the measurements, it is not possible to draw a firm conclusion as to whether or not orientation dependence is present. However, there seems to be a trend that the in-build plane hardness $(\mathrm{XY})$ is slightly higher than the across-plane (XZ and YZ), potentially due to a compounding effect of the bands shown above.

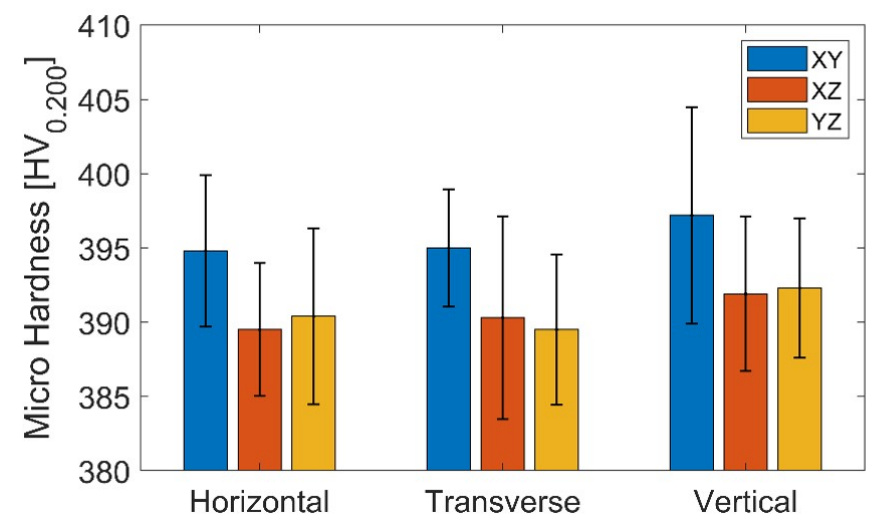

Figure 5: Micro hardness of tensile bars, measured on orthogonal planes XY, XZ and YZ.

Visually, the effect of the applied stress relief treatment has not been detected. In contrast, such an effect is observed in XRD (Figure 6). The only phase present in the diffractograms is martensite, $\alpha$, which cannot be distinguished from the $\alpha$ phase by diffraction. No $\beta$ peaks appear in this diffractogram, however as $\beta$ tends to be strongly textured, its actual presence cannot be excluded. The effect of stress relief is primarily indicated by the shift of the first two $\alpha$ peaks: 100 and 002. Most likely, the peak shift is due to a combination of stress relief and redistribution of the alloying elements through precipitation of nano-scale $\beta$ or intermetallics such as $\mathrm{Ti}_{3} \mathrm{Al}$, which was not detected by XRD. Particularly, the formation of $\beta$ would lower the V content in the $\alpha$ phase and thereby cause a peak shift to lower angles.

The bottom was observed to experience drastically different cooling conditions (Figure 1) and associated microstructure (Figure 4) as also seen in the diffractogram (Figure 6). The main differences are related to the first three $\alpha$ peaks, which are shifted to lower angles. A shoulder has developed at high-angle side the $101 \alpha$ peak, at the location of the $110 \beta$ peak. Therefore, it is concluded that the bottom consists of an $\alpha$ phase with a lower V content, causing the peak shift, and some $\beta$, which creates the shoulder. Furthermore, the peaks in the bottom experience broadening, which is most likely due to a variation in the chemical compositions of the phases in the bottom during cyclic heating. 

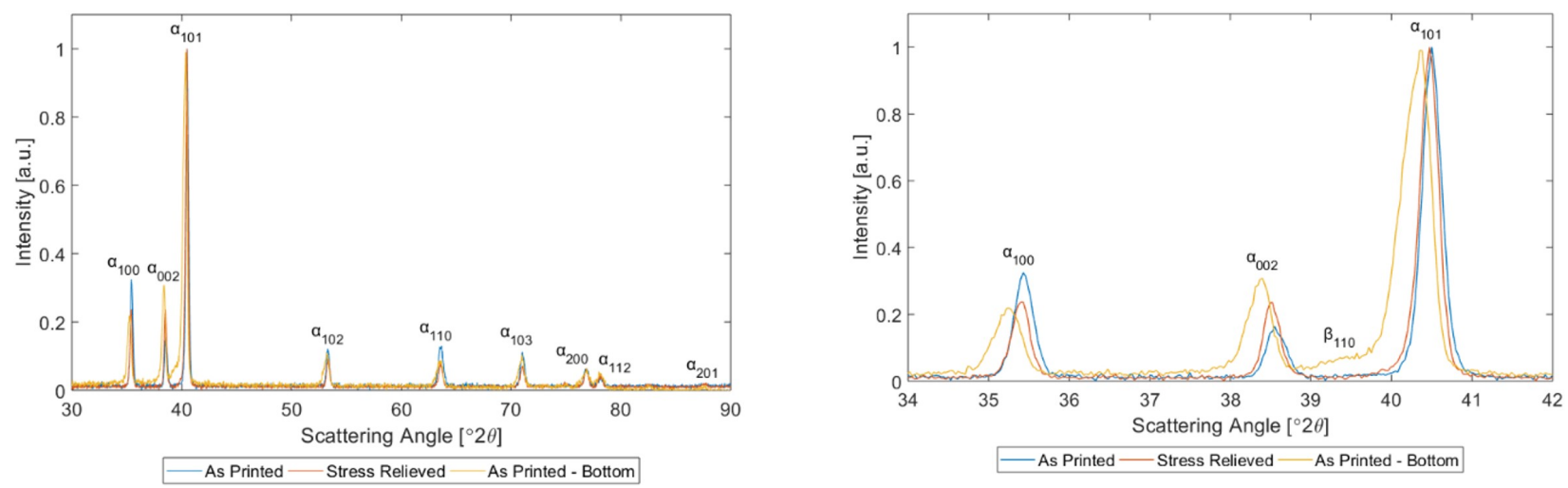

Figure 6: X-ray diffractograms for as-printed and stress relieved specimens.

The result of mechanical tensile testing of the specimens can be found in Table 2. The obtained Young's modulus (E), ultimate tensile strength (UTS) and yield strength $\left(\sigma_{\mathrm{y}}\right)$ are similar for samples of different orientations and they are also in accordance with the supplier's datasheet [9]. The obtained elongations show relatively large variation, both in the different orientations and among specimens of the same orientation, particularly the horizontal. This is consistent with earlier reports, where the transverse orientation provided the best ductility [8]. This is thought to be an effect of the orientation of the elongated grains with respect to the loading direction. Compared to the provided datasheet [9], the elongation is better than the expected 5\%, when the specimens are machined (see vertical and vertical rough results in Table 2). However, the rough surface finish clearly lowers the ductility from the expected level (from the data sheet [9]). The EDM treatment induces minimal mechanical deformation in the surface; however, there is an unknown thermal input. This could potentially also contribute to the improved ductility. Moreover, a rough surface finish is anticipated to have an even larger impact on the fatigue properties, which are sensitive to the surface conditions. Therefore, in most fatigue-loaded applications, the current as-printed condition will most likely be insufficient and require post processing.

Table 2: Result of tensile testing.

\begin{tabular}{|c|c|c|c|c||}
\hline & E [GPa] & UTS [MPa] & $\boldsymbol{\sigma}_{\mathbf{y}}$ [MPa] & Elongation [\%] \\
\hline Horizontal & $111 \pm 7$ & $1259 \pm 25$ & $1200 \pm 21$ & $7.4 \pm 2.7$ \\
\hline Transverse & $117 \pm 11$ & $1278 \pm 12$ & $1211 \pm 19$ & $10.2 \pm 1.4$ \\
\hline Vertical & $119 \pm 7$ & $1292 \pm 19$ & $1241 \pm 19$ & $8.3 \pm 1.1$ \\
\hline Vertical, rough & $119 \pm 10$ & $1283 \pm 4$ & $1226 \pm 011$ & $4.4 \pm 0.8$ \\
\hline \hline
\end{tabular}

No necking was visible on the tested specimens, indicating a uniform elongation. The fracture surfaces contain a mix of ductile and brittle fracture characteristics. Most fracture surfaces contained unmelted powder particles (Figure $7 \mathrm{~b}$ ). This indicates that the cavity associated with these particles may have acted as a crack nucleation point. Furthermore, many sites were observed where powder was located previously (Figure 7 a). Potentially, a heat treatment at sufficiently high temperature could sinter these particles and lower the density of crack nucleation sites, which would improve particularly the fatigue properties.

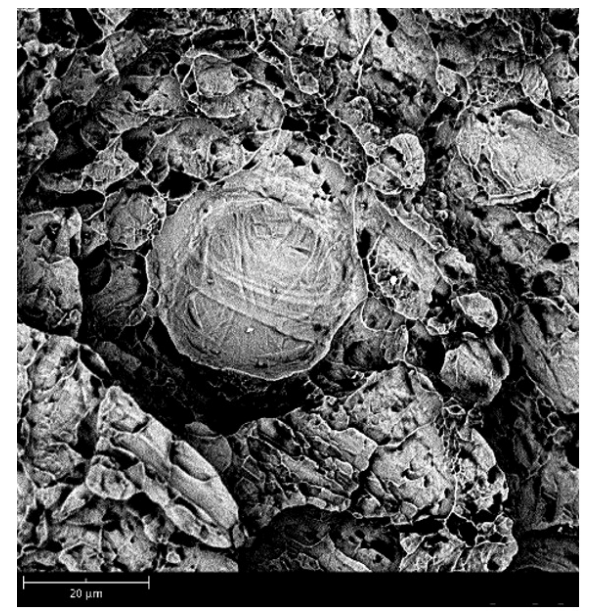

a)

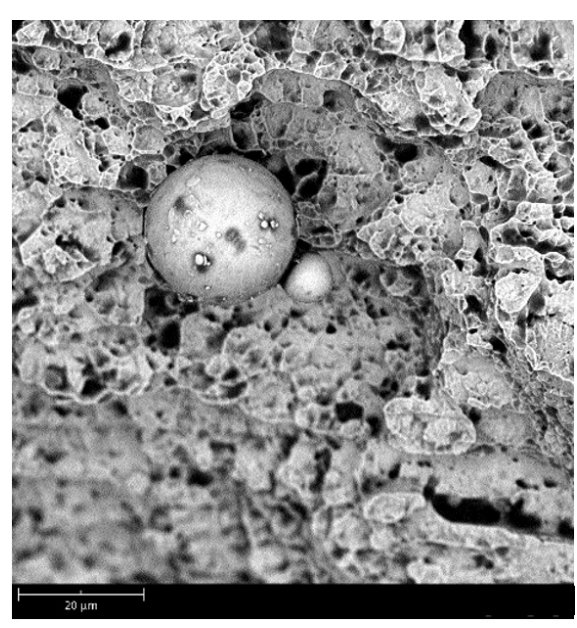

b)

Figure 7: SEM micrographs of fracture surfaces. a) location of removed particle and b) unmelted powder particles. 


\section{Conclusions}

The numerical modelling of the heat distribution in samples during SLM of tensile bars showed very different thermal histories, depending on the printing orientation and the specific location in the tensile bar. This, as expected, resulted in widely different microstructures in the bottom, bulk and top locations as the heat input was different here. The bulk consists of slightly elongated prior- $\beta$ grains with a fine martensitic structure. The bottom however, consists of a different microstructure, most likely $\alpha+\beta$ with alloying element partitioning in the phases. The ductility varied between the different orientations, with the transverse orientation exhibiting the highest elongation and the rough as-printed surface showing the lowest. Unmelted powder particles were found on the fracture surfaces and are held responsible for premature (low ductility) failure.

\section{Acknowledgements}

The authors thank the Innovation Fund Denmark for the funding for the AM-Line 2.0 project, including this research and Danish Technological Institute for providing specimens.

\section{References}

[1] S.L.N. Ford, Additive Manufacturing Technology: Potential Implications for U. S. Manufacturing Competitiveness, J. Int. Commer. Econ. (2014(30)), p. 1-35.

[2] T. DebRoy, H.L. Wei, J.S. Zuback, et al., Additive manufacturing of metallic components - Process, structure and properties, Prog. Mater. Sci. (2017, Volume 92), p. 112-224.

[3] L. Thijs, F. Verhaeghe, T. Craeghs, J. Van Humbeeck and J.P. Kruth, A study of the microstructural evolution during selective laser melting of Ti-6Al-4V, Acta Mater. (2010, Volume 58(9)), p. 3303-3312.

[4] T. Vilaro, C. Colin and J.D. Bartout, As-fabricated and heat-treated microstructures of the Ti-6Al-4V alloy processed by selective laser melting, Metall. Mater. Trans. A Phys. Metall. Mater. Sci. (2011, Volume 42(10)), p. 3190-3199.

[5] W. Xu, M. Brandt, S. Sun, et al., Additive manufacturing of strong and ductile Ti-6Al-4V by selective laser melting via in situ martensite decomposition, Acta Mater. (2015, Volume 85), p. 74-84.

[6] J. Mezzetta, J.P. Choi, J. Milligan, et al., Microstructure-Properties Relationships of Ti-6Al-4V Parts Fabricated by Selective Laser Melting, Int. J. Precis. Eng. Manuf. - Green Technol. (2018, Volume 5(5)), p. 605-612.

[7] ASTM-F2924, Standard Specification for Addtive Manufacturing Titanium-6 Aluminum-4 Vanadium with Powder Bed Fusion, 2014.

[8] M. Simonelli, Y.Y. Tse and C. Tuck, Effect of the build orientation on the mechanical properties and fracture modes of SLM Ti-6Al4V, Mater. Sci. Eng. A (2014, Volume 616), p. 1-11.

[9] SLM Solutions, 3D Metals, 2018. https:/www.slm-solutions.com/fileadmin/user_upload/200EN180924-02-POWDER_WEB.pdf 